\title{
Current management of type B aortic dissection
}

\author{
T Paul Tran' \\ Ali Khoynezhad ${ }^{2}$ \\ 'Department of Emergency Medicine; \\ ${ }^{2}$ Section of Cardiothoracic Surgery, \\ University of Nebraska Medical \\ Center, Omaha, NE, USA
}

\begin{abstract}
Acute aortic dissection is a life-threatening condition associated with high morbidity and mortality. In this article, the authors review basic biology of the aorta and aortic dissection, epidemiology, clinical presentation, diagnostic approach, emergency stabilization measures, and the latest surgical approach for type B dissection.
\end{abstract}

Keywords: acute aortic dissection, aorta, diagnosis, management, review

The tragedies of life are largely arterial.

- Sir William Osler ${ }^{1}$

\section{Perspective}

Diseases of the aorta are among the most feared conditions encountered in the emergency department. The term 'acute aortic syndrome' refers to a group of catastrophic diseases that affect the aorta. These include aneurysmal aortic rupture, intramural aortic hematoma, penetrating atherosclerotic ulcer, traumatic aortic transection, and acute aortic dissection (AAD).

Acute aortic dissection is not an infrequent clinical entity. With more than 20 cases per million per year, AAD is actually the most common catastrophe of the aorta. It is a time-sensitive and rapidly fatal disease: If left untreated, early mortality from type A AAD can be as high as $1 \%$ per hour, $50 \%$ at 72 hours, $75 \%$ at two weeks, and $90 \%$ at three months. ${ }^{2-5}$ While hourly mortality data for type B AAD are not available, the overall in-hospital mortality is reported to be $11 \%$. For those patients in the highest risk group, type B mortality can be as high as $71 \%{ }^{6}$

The classic clinical presentation of type B AAD is that of a male patient in his 60-70's with a history of hypertension, who presents to the emergency department (ED) with the complaint of abrupt onset of severe, sharp, ripping chest and/or back pain. Patient appears in distress with pain, sweating, and anxious as in shock. Blood pressure may be normal or elevated. Physical examination may reveal a pulse deficit, diastolic murmur, or focal neurological findings. Definitive imaging methods usually include a computed tomography (CTA) angiography. Critically ill patients will need definitive airway support, fluid resuscitation, blood pressure and heart rate control, and emergent open surgical or endovascular repair.

While type A AAD usually requires emergency surgical intervention, the majority of type B AAD (282 out of 386 [73\%] in a recent series by Suzuki) are managed medically. ${ }^{7}$ With advances in understanding of the pathogenesis and pathophysiology of $\mathrm{AAD}$, improved and aggressive antiimpulsive management, and the improvements in surgical/endovascular techniques, mortality from AAD has improved significantly. The aim of this manuscript is, first, to give an overview of aortic dissection and second, to focus on the contemporary approach to the diagnosis and medical management of type B acute aortic dissection. 


\section{Etiology and pathogenesis}

The heart serves as a positive displacement pump to supply blood to organs in humans. For every cardiac cycle, the left ventricle pushes (displaces) a fixed amount of blood into the aorta, which serves not only as a conduit but also as a modulator and damper to convert this pulsatile blood flow into a even reservoir of blood supply to the issues. The aortic wall is made up of three layers: the inner layer of intima, the middle layer of media, and the outer layer of adventitia (Figure 1). The intima is made up of an endothelial lining, a highly active metabolically monolayer, which is supported by a sublayer of loose connective tissue. This intimal sublayer allows the intima to move relative to the media when the aorta expands or contracts during systole and diastole.

The media is made up of a meshwork of concentric fenestrated lamellar layers of elastin fibers (about 50). Interposed between the elastin fiber layers are concentric smooth muscle cells and collagen fibers, proteoglycans, and glycoproteins. Elastin fibers are made of elastin, a highly stretchable material that is able to stretch up to $2-3 \mathrm{x}$ of its original length without rupture. Elastin is the material that gives the aorta its distensibility. Collagen fibers, on the other hand, are very stiff and are up to 5,000 times stiffer than elastin. Collagen gives the aorta the structural integrity and resistance to hemodynamic stress as well as the shearing effects created by the force of blood flow. In addition, the rings of smooth muscle cells in the media also contribute to aorta vascular stiffness. Under the influence of autonomic control, smooth muscle cells in the media contract and relax, giving the aorta its vascular tone.

The outermost layer, the adventitia, is made of the tough collagen fibers and connective tissue. Vasa vasorum ("vessels of the vessel") are small blood vessels located in the adventitia and the outer part the media. These blood vessels provide nutrients to the media and adventitia since the wall of the aorta is too thick to deride nourishment from the blood in the aortic lumen via diffusion.

Although it may appear overly simplistic, acute aortic dissection occurs when the aortic wall, already weakened by disease processes, is further subjected to elevated stress from systemic hypertension. Classic AAD begins with a tear in the intimal layer. Blood under pulsatile pressure subsequently forces the tear to open and dissects along the media layers in the diseased aortic wall, forming a false lumen(s) (Figure 2). The dissection can propagate distally, proximally, or both along the extent of the aorta. In the majority of cases $(>90 \%)$, as the dissection propagates, it also causes $\geq 1$ secondary tear in the intimomedial layer, allowing blood to flow back to the true lumen of the aorta. While the primary entry site is usually in the thoracic aorta, the secondary site can be in the lower descending or abdominal aorta. AAD can be catastrophic because the dissecting false lumen can rupture, causing rapid exsanguination and death. The false lumen can also obstruct the arterial branches coming off the aorta, causing serious and potentially lethal complications. In type A AAD, these include stroke, acute myocardial infarction, pericardial tamponade, or aortic valve insufficiency. In type B AAD, potential complications include intestinal ischemia, renal failure, paraplegia, and other malperfusion syndromes.

There are two similar pathological entities worth mentioning: intramural hematomas (IMH) and penetrating atherosclerotic ulcers (PAU). Patients with IMH or PAU have clinical presentations that are indistinguishable from AAD. IMH refers to a condition where there is a bleed within the aortic wall without the evidence of an intimomedial flap. It is often considered a precursor of the classic aortic dissection and thought to be the result of mechanical tearing of the vaso vasorum. The hematoma can expand inward causing a tear on the intimal side, causing blood to dissect along the extent of the aorta, resembling classic acute aortic dissection. PAU refers to a condition where there is an ulceration of atherosclerotic aortic plaques, usually in the descending thoracic and abdominal aorta. These lesions can lead to a penetration into the intimal borders and into the media, resembling a localized hematoma and an IMH. They can also start dissecting along the extent of the aorta, resembling a classic aortic dissection. The entry tear at the ulceration may be thicker and less mobile compared to the intimomedial flap seen in the classic aortic dissection. PAU tends to affect the descending thoracic aorta and the abdominal aorta. Its management is similar to type B AAD.

\section{Risk factors}

Both acquired conditions and connective tissue disorders are known to cause certain individuals to be at risk for acute aortic dissection. Data from the International Registry of Aortic Dissection (IRAD) ${ }^{6,8}$ showed the following risk factors in AAD: male sex, age, a history of hypertension or atherosclerosis, prior cardiac surgery including aortic valve surgery, a history of bicuspid aortic valve, or a history of Marfan syndrome. The average age for AAD was 63.1 years, with type B slightly older (66.3 vs 61.2 years). $65.3 \%$ of patients were male. $62.3 \%$ of the patients had type A dissection, $72.1 \%$ had a history of hypertension, and $4.9 \%$ had Marfan syndrome. ${ }^{6}$ Of particular interest to the physicians evaluating these patients in the emergency 


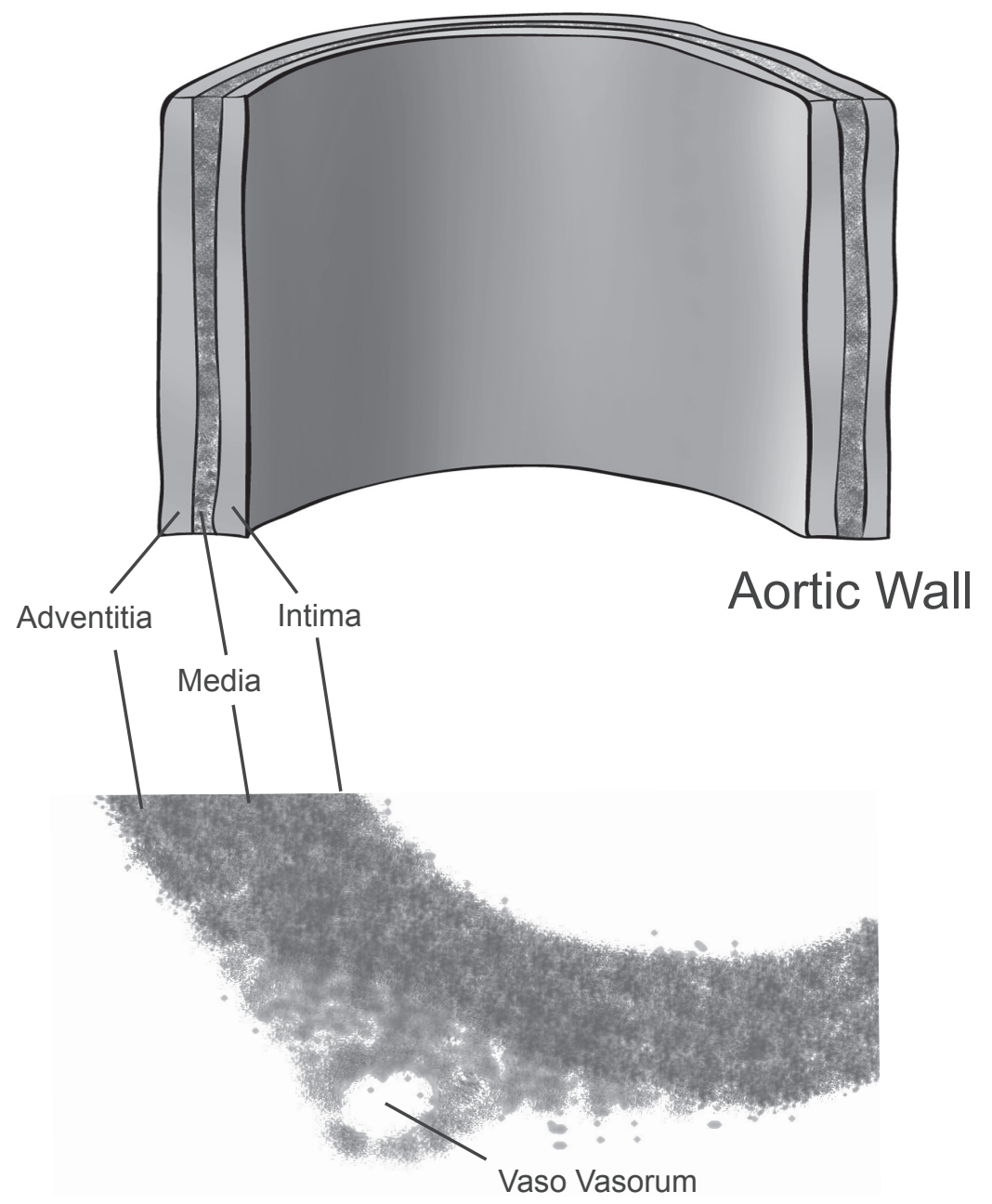

Figure I Layers of aortic wall.

department (ED), 7\% of patients presenting with AAD were younger than 40 years of age. Compared to the typical AAD patients, these younger patients were more likely to have Marfan syndrome, bicuspid aortic valve, Ehlers-Danlos syndrome, Loeys-Dietz syndrome and a history prior cardiac surgery. In the evaluation of acute chest pain syndrome in the younger patients, suspicious chest pain with the above characteristics should raise the index of suspicion for acute aortic dissection. Table 1 summarizes the distinguishing features in these two patient populations. ${ }^{8}$

\section{Vascular staging and classification}

Aortic dissection is categorized as acute or chronic depending on the onset of symptoms or dissection. If the symptoms or dissection occur within last 14 days, the dissection is termed acute aortic dissection (AAD). If the symptoms or dissection occur outside of the 14 days, the dissection is termed chronic aortic dissection. AAD is further classified depending on the location of the dissection. In the DeBakey classification (Figure 3A), type I refers to dissections that propagate from the ascending aorta, extend to the aortic arch, and commonly, beyond the arch distally. Type II refers to dissections that are confined to the ascending portion of the aorta. Type III dissections are limited to the descending aorta. Class III also includes the dissections that start in the descending aorta that extend proximally to the arch and ascending aorta. In the more commonly used Stanford classification system (Figure 3B), type A refers to dissections that involve the ascending and transverse aorta regardless of the site of origin. Type B refers to dissections that involve only the descending aorta. Rarely, dissections that start in the descending aorta can also extend proximally into the aortic arch and the ascending aorta. This is a special case of type B dissection, and should be called retro-A dissection.

The term "complicated" type B AAD is used for patients who present with, or during the hospital course, develop the following: rupture, malperfusion syndromes, 

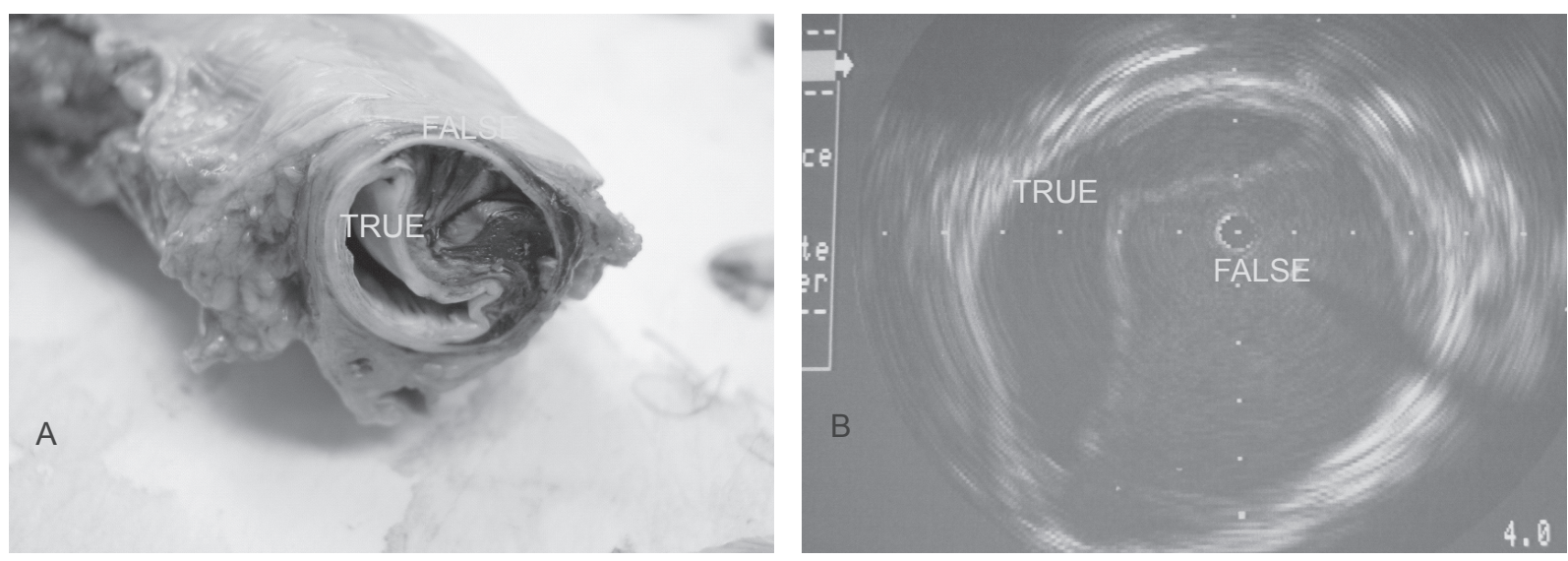

Figure 2 Aortic dissection. Actual specimen (A) and intravascular imaging (B) show both the true and false lumen.

refractory pain, or rapid aortic expansion. This is an important distinction as patients with complicated type B AAD usually require immediate endovascular or open surgical intervention.

\section{Clinical presentation}

Insights into the clinical presentation of patients are gained from the International Registry of Acute Aortic dissection (IRAD). ${ }^{6}$ A prototypical patient with acute aortic dissection will be a man in his fifth to seventh decade of life, whose past medical history is significant for hypertension; patients younger than 40 years may report a history of Marfan syndrome, bicuspid aortic valve, Ehlers-Danlos syndrome, Loeys-Dietz syndrome or aortic valve surgery. The most common chief complaint is sudden onset of severe chest pain or back pain. Patients with type A more commonly report a history of anterior chest pain whereas type B more commonly report a history of back or abdominal pain. The pain may be described as sharp, and, less commonly, ripping or tearing, with radiation to back, neck, or abdomen. One out of six

Table I Characteristics of patients $(N=95 I)$ with acute aortic dissection - from the International Registry of Acute Aortic Dissection $^{8}$

\begin{tabular}{|c|c|c|}
\hline Risk factor & $\begin{array}{l}\% \text { of Patients } \\
\text { Age }<40 \text { years }(N=68)\end{array}$ & $\begin{array}{l}\% \text { of Patients } \\
\text { Age } \geq \mathbf{4 0} \text { years }(N=883)\end{array}$ \\
\hline Age, years & 30.7 & 63.9 \\
\hline Type A dissection & 68 & 65 \\
\hline Type B dissection & 32 & 35 \\
\hline Male & 76 & 67 \\
\hline \multicolumn{3}{|l|}{ Medical and social history } \\
\hline Hypertension* & 34 & 72 \\
\hline Atherosclerosis* & I & 30 \\
\hline Known aortic aneurysm & 19 & 13 \\
\hline Bicuspid aortic valve* $(N=5 / 6)$ & 9 & 1 \\
\hline Known aortic valve disease & 10 & 8 \\
\hline Marfan syndrome* & 50 & 2 \\
\hline Peripartum & 3 & 0 \\
\hline Cocaine-related & 0 & 0.6 \\
\hline Diabetes mellitus & 0 & 4 \\
\hline \multicolumn{3}{|l|}{ Previous history of cardiac surgery } \\
\hline Aortic valve replacement* & 12 & 5 \\
\hline CABG & 0 & 6 \\
\hline Prior history of aortic dissection & 7 & 6 \\
\hline
\end{tabular}

Note: *Statistically different between the two groups. 


\section{Type I}

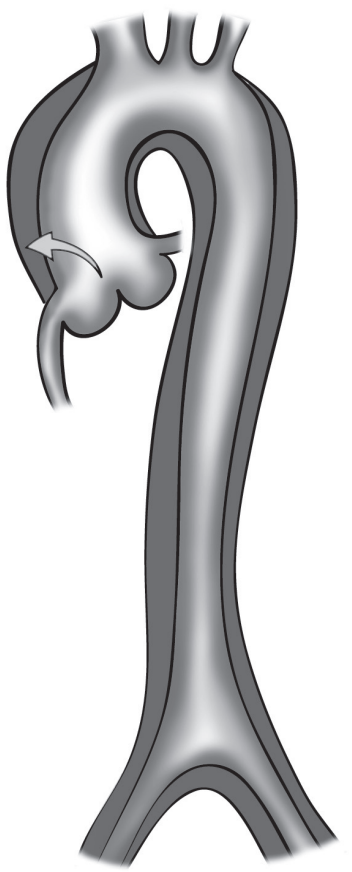

Type II

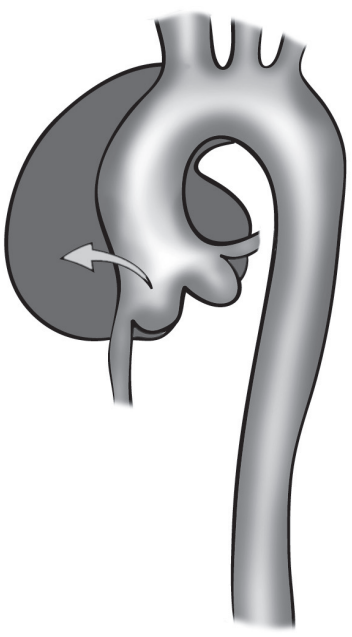

Type III

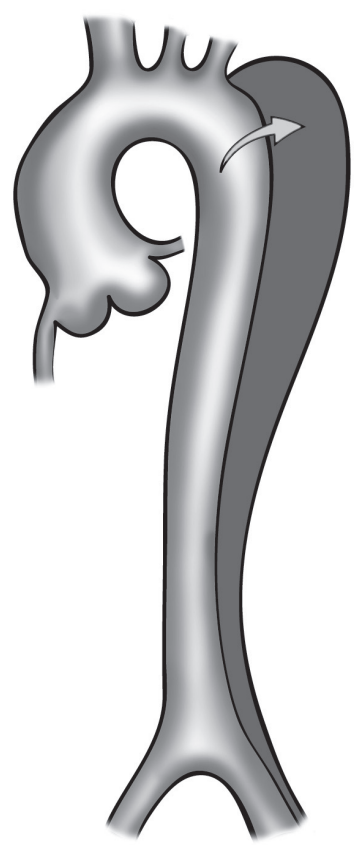

Figure 3A Debakey classification.

patients may ascribe a migratory quality to the pain ("I feel like the pain was going from my chest down to my legs"). The pain may wax and wane, sometimes with complete resolution of symptoms. In up to $4.5 \%$ of patients with aortic dissection, there was no pain reported. The absence of pain thus does not rule out an aortic dissection although it lowers the likelihood of a dissection (likelihood ratio, LR 0.3). ${ }^{9}$

In addition to the chest/back/abdominal pain, patients may present with generalized symptoms of dyspnea, diaphoresis, bloody diarrhea, nausea, and vomiting. These nonspecific symptoms, however, may be the prodrome of the ominous malperfusion syndrome. The clinical manifestation of the malperfusion syndrome depends on the anatomy of the affected vessels. Patients may present with signs or symptoms of a stroke, dizziness/syncope/aphasia/hemiplegia, if they have dynamic obstruction and malperfusion of coronary/ carotid/vertebral arteries. Alternatively, patients may complain of painful, plegic, pulseless, and cool extremities if the brachiocephalic, left subclavian arteries, distal aorta, iliac arteries are compromised. A dramatic paraplegia or quadriplegia is observed when the intercostal/segmental arteries are obstructed, resulting in reduced blood flow in the anterior spinal arteries and ischemic spinal cord. Abdominal pain and decreased urine output is a common malperfusion syndrome that results from obstruction of celiac trunk, superior mesenteric, inferior mesenteric, renal arteries causing (frequently fatal) acute bowel ischemia and decreased renal blood flow and glomerular filtration.

The physical examination overall is deemed not sufficiently sensitive to rule out an acute aortic dissection. ${ }^{9}$ However, the examination can be useful in raising the likelihood of an aortic dissection and whether a patient with a probable aortic dissection may need emergent surgical intervention. When performing the physical examination, the ED physician should focus on the assessment of hemodynamic stability, prognostic signs of poor outcome, and signs of impending cardiovascular collapse. The findings of pulse deficits, blood pressure differential of at least $20 \mathrm{~mm} \mathrm{Hg}$, or motor and sensory deficits on neurological examination significantly raise the likelihood ratio (LR 5.7) of an aortic dissection. ${ }^{9}$ A diastolic murmur of aortic regurgitation indicates possible involvement of the root of the ascending aorta. In a patient with hypotension, the presence of jugular venous distension or pulsus paradoxus indicates pericardial involvement and/or tamponade. These findings should prompt the clinician to seek emergent diagnostic confirmations and, at the same time, arrange for prompt cardiothoracic evaluation and intervention. 
Type A

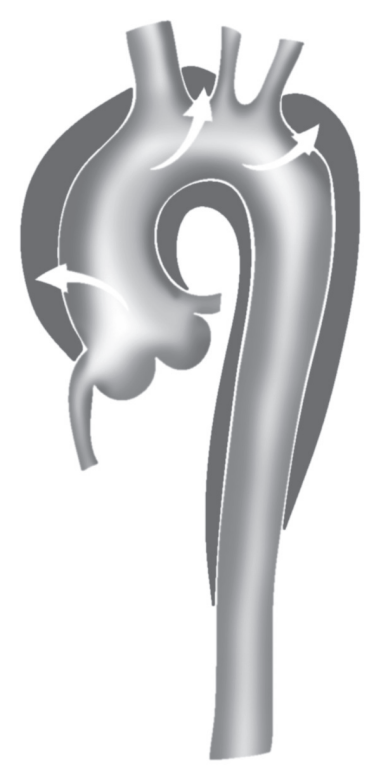

Type B

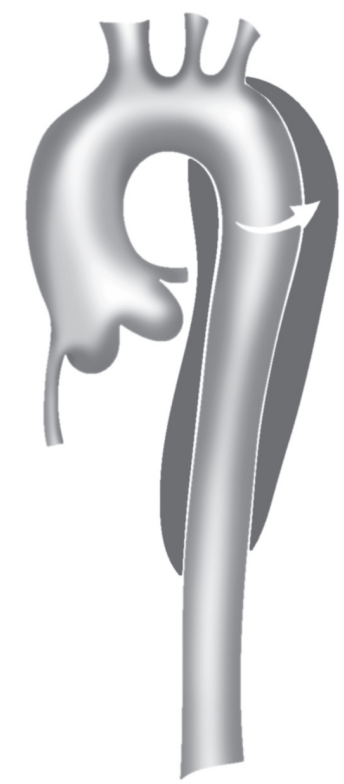

Figure 3B Stanford classification of aortic dissection. Stanford type A includes dissections that involve the ascending aorta, arch, and descending thoracic aorta. Stanford type B includes dissections that originate in the descending (and thoracoabdominal) aorta, regardless of any retrograde involvement of the arch.

\section{Diagnostic strategy and management}

Timely and accurate diagnosis of acute aortic dissection in the ED remains a clinical challenge. Of the approximately 4.4 million patients who present annually to the US ED's for chest pain, only about 2,000 have AAD. ${ }^{9,10}$ Partly as a result, $\mathrm{AAD}$ is only correctly diagnosed in $15 \%-43 \%$ of patients in the initial presentation. ${ }^{11,12}$ A somewhat more humbling fact is that up to $53 \%$ of cases of AAD were diagnosed after patients have been in the hospital for more than 24 hours. ${ }^{13}$

History and physical examination alone are not sufficiently sensitive to rule out an AAD. Although definitive imaging tools are available to rule out an acute aortic syndrome, indiscriminate use of invasive diagnostic tools in patients with low pre-test probabilities will inevitably lead to false positives, and in turn, higher health care costs, unnecessary anxiety, and potential complications for patients. $\mathrm{AAD}$, however, carries a mortality rate of $1 \%-2 \%$ per hour if unrecognized or not treated promptly. The human toll and medical legal implications for failures to diagnose an acute aortic dissection promptly are well publicized. ${ }^{14}$ The strategy of deferring the workup to the consultants or the admitting service is unacceptable. ${ }^{15}$ In approaching patients with acute chest pain in the ED, physicians need to methodically rule out the life threatening causes, ascertain the diagnosis, and initiate the stabilization measures. These life threatening causes include acute aortic syndrome, acute coronary syndrome, pulmonary embolus, tension pneumothorax, and esophageal rupture.

The evaluation should begin with an immediate assessment of vital signs and hemodynamic status. If patients are critically unstable, ACLS protocol should immediately be started. Patients should be placed on a monitor, have two large bore ( $>18$ gauge) intravenous access, and oxygen administered. An electrocardiogram (EKG) should be performed within 10 minutes of patient's arrival. A portable chest X-ray should also be done concurrently with other stabilization measures. In patients at high risks for aortic dissection, 10 units of red blood cells should be typed and crossed.

In the setting of chest or back pain, the presence of a pulse differential between carotid, radial, or femoral arteries strongly suggests the diagnosis of acute aortic dissection (LR 5.7). ${ }^{9}$ The presence of focal neurological deficits such as transient ischemic attack or hemiplegia even more strongly suggests the possibility of an aortic dissection. The likelihood ratio for aortic dissection among patients presenting with focal neurological deficits range from 6.6 to $33 .{ }^{9}$ Chest X-rays findings classically associated with aortic dissection include abnormal aortic contour, pleural effusion, displaced intimal calcification, and wide mediastinum. Although these classic radiographic findings are not always present, collectively, up to $90 \%$ of patients with aortic dissection proved to have some abnormal findings on chest X-rays. Absence of wide mediastinum and abnormal aortic contour on chest X-ray lowers the likelihood of aortic dissection (LR 0.3). ${ }^{9}$

\section{Biochemical markers}

The Holy Grail in the workup of acute aortic dissection is an inexpensive bedside blood test with sufficient sensitivity (good negative predictive value) that physicians can use to rapidly rule out an acute dissection. Of the several biochemical markers that were evaluated in the last decade, the following three assays deserve further discussion.

Smooth muscle myosin heavy chain, a major component of the smooth muscle in the aortic medial layer, is released to the circulation shortly after the onset of dissection. In a pilot study, the assay $(>2.5$ microgram $/ \mathrm{L})$ had a sensitivity of $90.9 \%$ and specificity of $98 \%$ in detecting acute aortic dissection as compared to healthy volunteers. ${ }^{16}$ These test numbers are, however, limited to patients with proximal dissection who present within 3 hours of onset of symptoms. 
Elastin is another major structural component of the medial layer of aortic wall. In a retrospective study using the assay for soluble elastin fragments (sELAF) that was developed by the authors, ${ }^{17}$ Shinohara and colleagues demonstrated that an ELISA measuring sELAF in the serum with the cutoff set at $+3 \mathrm{SD}$ (standard deviation) above the mean of age-adjusted healthy subjects had a sensitivity of $88.9 \%$ $(16 / 18)$ and specificity of $99.8 \%(473 / 474) .{ }^{17}$ The positive (PPV) and negative predictive values (NPV) were 94.1\% and $98.8 \%$, respectively. Based on the NPV, measuring sELAF would appear like an excellent test to rule out an acute aortic dissection. However, since this study was retrospective, data therein need to be confirmed in a prospective confirmatory multicenter study. More importantly, the ELISA for sELAF takes 3 hours to perform, a major drawback for a time sensitive condition such as acute aortic dissection. A positive test would require a follow-up radiologic confirmatory test, which further prolongs the diagnosis.

Plasma D-dimer, a degradation product of cross-linked fibrin, has emerged as a promising diagnostic marker with high sensitivity for exclusion of acute aortic dissection. The rise in D-dimer is thought to result from the thrombogenicity of the false lumen in $\mathrm{AAD}$ and the subsequent activation of the coagulation cascade. As such, D-dimer values are expected to be lower in proximal AAD (type A). In an earlier pooled analysis by Sodeck, a d-dimer $<0.1 \mu \mathrm{g} / \mathrm{ml}$ is though to exclude all AAD (sensitivity of $100 \%) .{ }^{18} \mathrm{In}$ a more recent pooled sensitivity analysis in EM literature, using a commonly used threshold for a positive D-dimer test of $<0.5 \mu \mathrm{g} / \mathrm{ml}$, Marill found that serum d-dimer had a sensitivity of $94 \% .{ }^{19}$ The authors concluded that serum d-dimer can be used in patients with a low likelihood of AAD. Overall, a preponderance of recent studies support drawing D-dimer in the ED given its high sensitivity, noninvasiveness, broad availability and low cost. ${ }^{20-24}$

\section{Imaging}

The choice for the diagnostic imaging depends on patient's stability, local expertise, and availability. The main goals in diagnostic imaging are to rapidly confirm (or exclude) an AAD, classify the type/extent of the AAD, identify and locate the intimal tears, confirm the presence of true/false lumen and whether a thrombus is present, assess any aortic side branch involvement, detect any aortic regurgitation, and discover any extravasation to the pericardium, mediastinum, or thoracic cavity.

A chest X-ray can detect abnormalities in 60\%-90\% of cases of AAD. Abnormalities that may suggest an AAD include an abnormal aortic knob, deviation of the trachea, main stem bronchus, or esophagus, wide mediastinum, pleural cap, or pleural effusion. A normal chest X-ray, however, can not be used to rule out an AAD. In unstable patients with strong suspicion of an AAD, chest X-rays may lead to further delay to definitive imaging and therapy and should be avoided.

Definitive imaging techniques are essential in the workup of AAD. Data from the 2002 IRAD show that computed tomography angiography (CTA) is used in $63 \%$ of cases of suspected AAD, followed transesophageal echocardiography (TEE) in 32\%, aortography $4 \%$, and magnetic resonance angiography (MRA) in $1 \% .{ }^{25}$ Compared with the newer imaging techniques, aortography has a number of serious disadvantages, including the use of a heavy dose of IV contrast $(1 \mathrm{mg} / \mathrm{kg})$, the risks of an invasive procedure, and the extended time it takes to complete the procedure (up to $2^{+}$hours). As a result, this imaging method is rarely the first diagnostic modality in the workup of AAD.

Computed tomography angiography, TEE and MRA have similar pooled sensitivity (98\%-100\%) and specificity (95\%-98\%), ${ }^{26}$ although the pooled positive likelihood ratio appeared to be higher for MRA (positive likelihood ratio, $25.3 ; 95 \%$ confidence interval, 11.1-57.1) than for TEE (14.1; 6.0-33.2) or CTA $(13.9 ; 4.2-46.0)$. CTA is widely available and relatively rapid, provides visualization of the entire aorta down to iliac arteries, and delineates the involvement of aortic side branches. It is usually the first choice for imaging in the work up of AAD. Disadvantages with CTA include the requirement that patients be transported to the CTA suite, the use of potentially nephrotoxic contrast, and the inability to assess aortic insufficiency.

Overall, CTA of the chest is the preferred first diagnostic imaging method in hemodynamically stable patients (Figure 4). The choice for a second imaging study includes MRA or TEE, depending on clinical condition and local environment. MRA is highly accurate and does not require the use of a contrast dye. It is, however, usually not available on an emergency basis and requires patients to be in MRA suite for an extended period of time. Other issues such as claustrophobia, the use of ventilator, and patient's use of metal devices (pacemakers, aneurysmal clips) may further complicate its routine use. ${ }^{25}$ TEE is a viable alternative in patients who are critically ill and/or hemodynamically unstable. The main advantages of a TEE include speed, good sensitivity and specificity, and the fact that it can be performed at the patient's bedside in the ED. Its main limitations are lack of widespread expertise and 24/7 availability. 


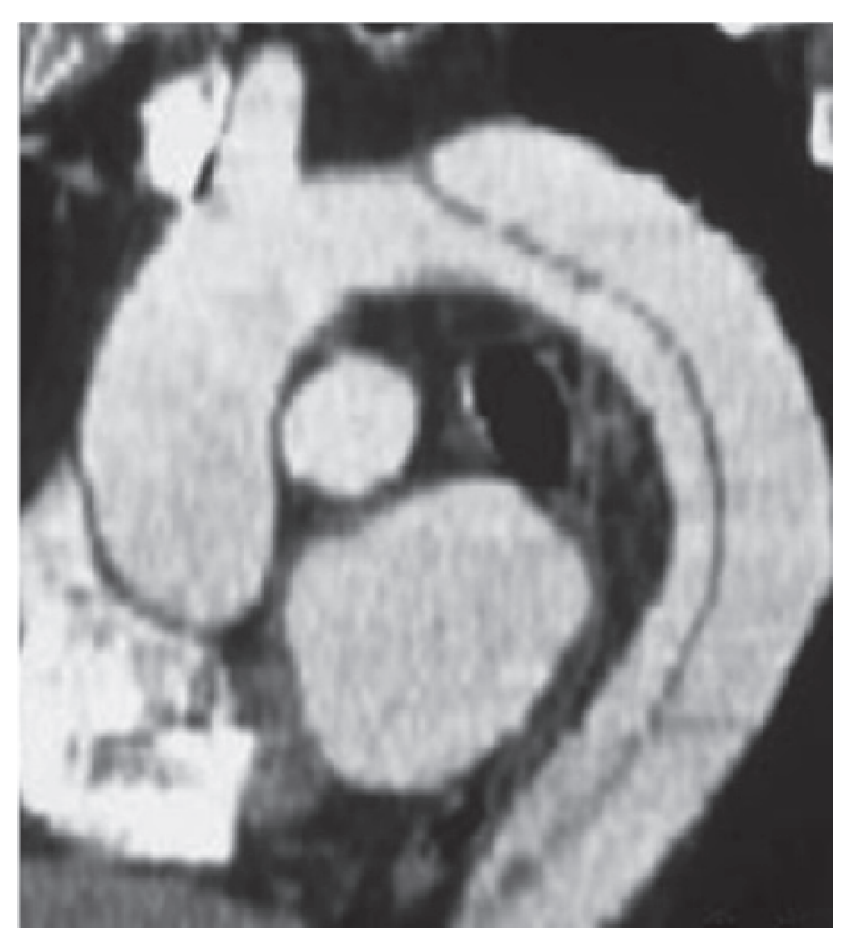

Figure 4 Computer tomography with enhanced contrast showing type B dissection.

If the diagnosis can not be established using the aforementioned imaging modalities, the patient should be taken to the hybrid operating room. An intravascular ultrasound and/or aortography performed by the cardiothoracic surgeon will establish the definitive diagnosis (similar to diagnostic algorithm of traumatic aortic transection). ${ }^{27}$

Intravascular ultrasound (IVUS) is a novel approach in diagnosis of type B AAD that has the highest accuracy of any modality (Figure 5). IVUS has been demonstrated $100 \%$ sensitivity and specificity during endovascular diagnosis and treatment of complicated type B aortic dissection. ${ }^{28}$ Furthermore, IVUS is a critical imaging modality for endovascular treatment options for type B AAD. ${ }^{29}$

\section{Management}

The modern surgical treatment for AAD, including those for acute type $\mathrm{B}$ aortic dissection, began after the landmark aortic operation by Drs Cooley and DeBakey in the 1950's. ${ }^{30}$ In the decades following this sentinel event, patients with type $\mathrm{B}$ aortic dissection were mostly treated surgically. The medical management of acute type B AAD began to gain credence with the concept of antiimpulsive therapy as described by Wheat. ${ }^{31} \mathrm{He}$ was one of the first to demonstrate the importance of the force of contraction $\left(\mathrm{dP}^{\mathrm{d} \mathrm{dt}_{\text {max }}}\right)$ and blood pressure in the propagation of AAD in a dog model. ${ }^{32}$ Starting in the early 70 's, medical management of uncomplicated

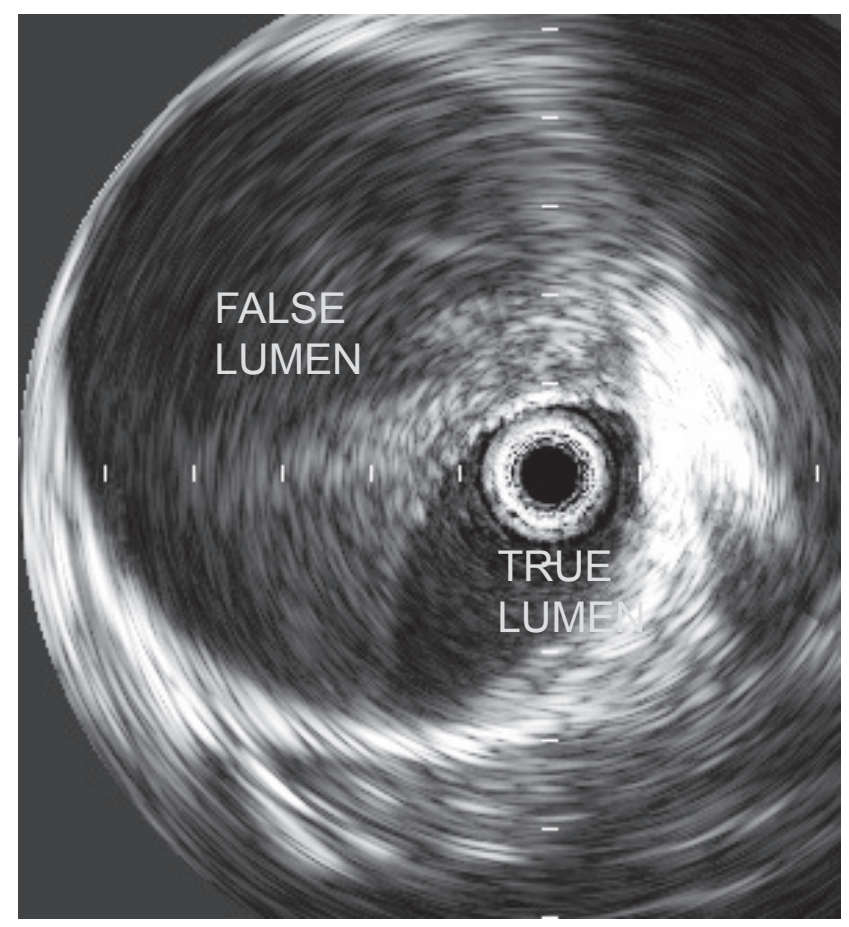

Figure 5 Intravascular imaging of aortic dissection.

type B AAD increasingly became the standard of care due to availability of potent beta-blockers and the lower mortality compared to surgical approach (for type A, early mortality rates of $10 \%$ vs. $35 \%$, respectively). ${ }^{33}$ Today, the antiimpulsive and antihypertensive combination therapy remains the cornerstone of modern medical management of type B AAD. Table 2 illustrates the authors' approach to the diagnosis and management of an acute aortic dissection (AAD) in the ED.

At triage, patients who present to the ED with sudden onset of chest, back, or abdominal pain should be quickly brought to a room to be examined by a physician. Prompt diagnosis and treatment of patients with suspected AAD is essential for improved outcome. The abbreviated history and physical examination should focus on time of onset, risk factors for aortic dissection, and findings that are consistent with AAD (Table 2). Patients should have IV's and A-line established, supplemental oxygen, be put on a cardiac monitor, an EKG, portable chest X-ray, blood draws, and be typed and crossed for 10 units of PRBC's. Surgical consultation should be initiated as soon as the emergency physician is comfortable with his/her clinical impression.

Pain should be treated with appropriate analgesics (eg, morphine). Heart rate and hypertension should be aggressively controlled with $\beta$-blockers. ${ }^{34}$ An arterial 
Table 2 Approach to acute aortic dissections (AAD) in the emergency department

I) Have a high index of suspicion for AAD

a) History:

i) Sudden onset, severe, sharp or tearing back pain, chest pain, shoulder pain, or abdominal pain

ii) Older than 60 years, history of hypertension, aortic dissection or aortic aneurysm (of family history of such), previous cardiac surgery, connective tissue disorder (Bicuspid aortic valve, Marfan syndrome, Ehler-Danlos syndrome, Loeys-Dietz syndrome), or peripartum

b) Physical examination:

(I) Pulse deficit, blood pressure differential in various extremities

(2) Neurological deficits

(3) Abdominal pain, flank pain

2) General measures:

a) Establish two large bore (>I8gauge) IV's

b) Administer supplemental oxygen by nasal cannula or nonrebreather mask

c) Put patient on cardiac monitor

d) Get an EKG, portable chest X-ray, place a Foley catheter

e) Obtain CBC, chemistry panel, coagulation panel, UA, CK, Troponin, d-dimer

f) Type and cross 10 units packed red blood cells (PRBC's)

g) Set up an arterial line

3) Early cardiothoracic surgical consultation

4) Definitive imaging:

a) Computed tomography angiogram (CTA)

b) Transesophageal echocardiogram

c) Magnetic resonance angiogram (MRA)

d) Intravascular ultrasound

e) Aortography

5) Blood pressure, heart rate, and pain management

a) First line: $\beta$-blockers

i) Labetalol, bolus ( $15 \mathrm{mg}) \pm$ a drip ( $5 \mathrm{mg} /$ hour $)$,

b) If hypertension persists, add:

i) Nicardipine drip (starting dose: $5 \mathrm{mg} / \mathrm{h}$ )

c) If tachycardia persists, add:

i) Esmolol (loading $0.5 \mathrm{mg} / \mathrm{kg}$ over $2-5 \mathrm{~min}$, followed by a drip of $10-20 \mu \mathrm{g} / \mathrm{kg} / \mathrm{min}$ )

ii) Diltiazem drip (loading $0.25 \mathrm{mg} / \mathrm{kg}$ over $2-5 \mathrm{~min}$, followed by a drip of $5 \mathrm{mg} / \mathrm{h}$ )

d) Goals:

(I) Heart rate $<60$ beats/min

(2) Systolic blood pressure $<100 \mathrm{~mm} \mathrm{Hg}$

e) Morphine (for pain relief)

6) Hemodynamically unstable patients

a) Tracheal intubation, mechanical ventilation

b) Blood pressure support with crystalloid and colloid (PRBC's if rupture is suspected)

c) TEE at bedside in the Emergency Department or in the OR

d) Pericardiocentesis is not recommended (class III)

vasodilator without the reflex tachycardia (eg, nicardipine), can be added as second line drugs for refractory hypertension. $\beta$-blockers are the first line drugs because they control the maximal force of left ventricular contraction $\left(\mathrm{dP} / \mathrm{dt}_{\max }\right)$ in addition to controlling HR and BP. This helps reduce further dissection, branch-vessel malperfusion and weakening of the aortic wall. Common $\beta$-blockers include labetalol, esmolol, metoprolol, and atenolol. Targeted HR and systolic BP should be $<60$ beats/min and $<100 \mathrm{~mm} \mathrm{Hg}$, respectively. In patients with potential intolerance to $\beta$-blockers (eg, asthma, heart failure), a test dose of a short acting $\beta$-blocker such as esmolol should be tried. ${ }^{35}$ If $\beta$-blockers can not be used, calcium channel blockers (eg, nicardipine, clevidipine or diltiazem) might be alternatives to control blood pressure in these patients. Hydralazine and sodium nitroprusside have been used in the past for medical management of type B AAD. Although these agents reduce blood pressure, they increase the maximal force of left ventricular contraction $\left(\mathrm{dP}^{\mathrm{dt} \mathrm{dt}_{\max }}\right)$ and cause reflex tachycardia and are, thus, contraindicated in patients with AAD.

Hemodynamically unstable patients with type B AAD are either in hemorrhagic shock or septic/metabolic shock from visceral or limb malperfusion. They should be endotracheally intubated and fluid resuscitated with crystalloid, PRBC's, or other colloid. In these patients, a TEE or CTA are the preferred diagnostic tools, depending on the hospital setting. 
If the diagnosis of a complicated type B AAD is confirmed, patients should be immediately brought to a hybrid operating room for endovascular and/or surgical treatment options. Endovascular or surgical options may be offered to hemodynamically stable patients with a complicated type B AAD. Table 3 lists the indications for endovascular or surgical intervention in patients with type B AAD.

\section{Outcome}

The short term prognosis for patients with type B AAD is better than those with type A and the medical management of type B AAD is associated with less mortality compared to the open surgical intervention approach. Overall, 89\% of patients with type B AAD survive to hospital discharge, although the in-hospital survival rates were as low as $29 \%$ for the highest risk group, $64 \%$ for the intermediate, and $97 \%$ for the lowest risk group. ${ }^{6,36}$ Aortic rupture, shock, and malperfusion are the most important in-hospital risks leading to poor outcome in up to $20 \%$ of these patients. ${ }^{6,35}$ Of survivors, $80 \%$ will develop aneurysmal dilatation of the false lumen, requiring cardiothoracic surgical intervention in one third of the cases. ${ }^{35,37,38}$ Therefore, although medical management of patients with type B AAD is the standard of care at the moment, it is far from being an option with acceptable results to patients with uncomplicated type B AAD.

The long term prognosis for patients with type B AAD is poor. Data from IRAD indicated about 1 out of 4 patients with type $B$ dissection died at the 3-year mark regardless of the mode of therapy. The 3 -year rates of survival were $77.6 \%$, $82.8 \%$, and $76.2 \%$ for patients treated medically, surgically, and with endovascular therapy, respectively. ${ }^{36}$ Umana and colleauges reported the outcome of 189 patients with type $\mathrm{B}$ aortic dissection over a 36-year period. Actuarial survival for all patients was $71 \%, 60 \%, 35 \%$ and $17 \%$ at one, five, 10 , and 15 years regardless of medical or surgical therapy. ${ }^{33}$ A diameter $>4 \mathrm{~cm}$, patent false lumen, and a partially thrombosed false lumen are independent predictors of poor long term outcome for type B dissection. ${ }^{39,40}$

Table 3 Indications for endovascular or surgical intervention in patients with type B AAD

- Persistent or recurrent pain despite adequate antiimpulsive and antihypertensive therapy (at least two parenteral agents at moderate to high dose)

- Acute expansion of the false lumen

- Periaortic or mediastinal hematoma (contained rupture)

- Visceral, renal or limb malperfusion syndrome

Neurological deficits (relative indication).

\section{Future development}

Given the disappointing results of medical and surgical therapy in uncomplicated type B AAD, there has been interest in use of endovascular stenting along with antiimpulsive and antihypertensive treatment. The endovascular therapy is based on the concept that obliteration and thrombosis of the false lumen may result in improved long-term outcome. The INSTEAD trial was designed to address this question, but was associated with methodological flaws by including patients with chronic type B aortic dissection. ${ }^{41}$ Ongoing and future trials will shed light on this issue. For complicated type B AAD, endovascular therapies are becoming the standard of care in the Centers of Excellence, because they have shown to have a better outcome compared to the open repair approach. ${ }^{29,42,43}$

\section{Disclosure}

The authors report no conflicts of interest in this work.

\section{References}

1. Osler W, Silverman ME, Murray TJ, et al. American College of Physicians. The quotable Osler. Philadelphia: American College of Physicians, 2003.

2. Coady MA, Rizzo JA, Goldstein LJ, et al. Natural history, pathogenesis, and etiology of thoracic aortic aneurysms and dissections. Cardiol Clin. 1999; 17:615-35.

3. DeBakey ME, McCollum CH, Crawford ES, et al. Dissection and dissecting aneurysms of the aorta: twenty-year follow-up of five hundred twenty-seven patients treated surgically. Surgery. 1982;92:1118-34.

4. Fuster V, Andrews P. Medical treatment of the aorta. I. Cardiol Clin. 1999;17:697-715, viii.

5. Lindsay J, Jr., Hurst JW. Clinical features and prognosis in dissecting aneurysm of the aorta. A re-appraisal. Circulation. 1967;35:880-8.

6. Hagan PG, Nienaber CA, Isselbacher EM, et al. The International Registry of Acute Aortic Dissection (IRAD): new insights into an old disease. JAMA. 2000;283:897-903.

7. Suzuki T, Mehta RH, Ince H, et al. Clinical profiles and outcomes of acute type $\mathrm{B}$ aortic dissection in the current era: lessons from the International Registry of Aortic Dissection (IRAD). Circulation. 2003;108(Suppl 1):II312-7.

8. Januzzi JL, Isselbacher EM, Fattori R, et al. Characterizing the young patient with aortic dissection: results from the International Registry of Aortic Dissection (IRAD). J Am Coll Cardiol. 2004;43:665-9.

9. Klompas M. Does this patient have an acute thoracic aortic dissection? JAMA. 2002;287:2262-72.

10. McCaig LF, Nawar EW. National Hospital Ambulatory Medical Care Survey: 2004 emergency department summary. Adv Data. 2006;(372):1-29.

11. Meszaros I, Morocz J, Szlavi J, et al. Epidemiology and clinicopathology of aortic dissection. Chest. 2000;117:1271-8.

12. Sullivan PR, Wolfson AB, Leckey RD, et al. Diagnosis of acute thoracic aortic dissection in the emergency department. Am J Emerg Med. 2000;18:46-50.

13. Viljanen T. Diagnostic difficulties in aortic dissection. Retrospective study of 89 surgically treated patients. Ann Chir Gynaecol. 1986;75:328-32.

14. The Associated Press. Winkler Testifies in John Ritter Lawsuit. The New York Times. 2008; Feb 13. 
15. Boie ET. Initial evaluation of chest pain. Emerg Med Clin North Am. 2005;23:937-57.

16. Suzuki T, Katoh H, Tsuchio Y, et al. Diagnostic implications of elevated levels of smooth-muscle myosin heavy-chain protein in acute aortic dissection. The smooth muscle myosin heavy chain study. Ann Intern Med. 2000;133:537-41.

17. Shinohara T, Suzuki K, Okada M, et al. Soluble elastin fragments in serum are elevated in acute aortic dissection. Arterioscler Thromb Vasc Biol. 2003;23:1839-44.

18. Sodeck G, Domanovits H, Schillinger M, et al. D-dimer in ruling out acute aortic dissection: a systematic review and prospective cohort study. Eur Heart J. 2007;28:3067-75.

19. Marill KA. Serum D-dimer is a sensitive test for the detection of acute aortic dissection: A pooled meta-analysis. J Emerg Med. 2008;34:367-76.

20. Eggebrecht H, Naber CK, Bruch C, et al. Value of plasma fibrin D-dimers for detection of acute aortic dissection. $\mathrm{J} \mathrm{Am} \mathrm{Coll} \mathrm{Cardiol.}$ 2004;44:804-9.

21. Hazui H, Fukumoto H, Negoro N, et al. Simple and useful tests for discriminating between acute aortic dissection of the ascending aorta and acute myocardial infarction in the emergency setting. Circ $J$. 2005;69:677-82.

22. Immer FF. Is there a place for D-dimers in acute type A aortic dissection? Heart. 2006;92:727-8.

23. Ohlmann P, Faure A, Morel O, et al. Diagnostic and prognostic value of circulating D-Dimers in patients with acute aortic dissection. Crit Care Med. 2006;34:1358-64.

24. Ohlmann P, Morel O, Radulescu B, et al. D-Dimer in ruling out acute aortic dissection: sensitivity is not $100 \%$. Eur Heart J. 2008;29:828-9; author reply 829 .

25. Moore AG, Eagle KA, Bruckman D, et al. Choice of computed tomography, transesophageal echocardiography, magnetic resonance imaging, and aortography in acute aortic dissection: International Registry of Acute Aortic Dissection (IRAD). Am J Cardiol. 2002;89:1235-8.

26. Shiga T, Wajima Z, Apfel CC, et al. Diagnostic accuracy of transesophageal echocardiography, helical computed tomography, and magnetic resonance imaging for suspected thoracic aortic dissection: systematic review and meta-analysis. Arch Intern Med. 2006;166:1350-6.

27. Khoynezhad A, Donayre CE, Kopchok GE, et al. Use of intravascular ultrasound in endovascular stenting of traumatic rupture of the descending thoracic aorta. Cardiothoracic Surgical Network (CTSNet) [serial on the internet]. 2006 Jun. [cited 2008 Jul 22]. Available from http://www. ctsnet.org/sections/clinicalresources/clinicalcases/article-12.html

28. Lee JT, White RA. Basics of intravascular ultrasound: an essential tool for the endovascular surgeon. Semin Vasc Surg. 2004;17:110-8.
29. Khoynezhad A, Donayre CE, Kopchok GE, et al. Mid-term results of endovascular treatment of complicated acute type B aortic dissection. J Thorac Cardiovasc Surg. 2008;136:424-30.

30. DeBakey ME, Beall AC Jr., Cooley DA, et al. Dissecting aneurysms of the aorta. Surg Clin North Am. 1966;46:1045-55.

31. Wheat MW Jr., Palmer RF, Bartley TD, et al. Treatment of dissecting aneurysms of the aorta without surgery. J Thorac Cardiovasc Surg. 1965;50:364-73.

32. Prokop EK, Palmer RF, Wheat MW Jr. Hydrodynamic forces in dissecting aneurysms. In-vitro studies in a Tygon model and in dog aortas. Circ Res. 1970;27:121-7.

33. Umana JP, Lai DT, Mitchell RS, et al. Is medical therapy still the optimal treatment strategy for patients with acute type B aortic dissections? J Thorac Cardiovasc Surg. 2002;124:896-910.

34. Khoynezhad A, Plestis KA. Managing emergency hypertension in aortic dissection and aortic aneurysm surgery. J Card Surg. 2006;21(Suppl 1):S3-S7.

35. Erbel R, Alfonso F, Boileau C, et al. Diagnosis and management of aortic dissection. Eur Heart J. 2001;22:1642-81.

36. Tsai TT, Fattori R, Trimarchi S, et al. Long-term survival in patients presenting with type B acute aortic dissection: insights from the International Registry of Acute Aortic Dissection. Circulation. 2006;114:2226-31.

37. Fann JI, Smith JA, Miller DC, et al. Surgical management of aortic dissection during a 30-year period. Circulation. 1995;92(9 Suppl): II113-21.

38. Juvonen T, Ergin MA, Galla JD, et al. Prospective study of the natural history of thoracic aortic aneurysms. Ann Thorac Surg. 1997;63:1533-45.

39. Onitsuka S, Akashi H, Tayama K, et al. Long-term outcome and prognostic predictors of medically treated acute type B aortic dissections. Ann Thorac Surg. 2004;78:1268-73.

40. Tsai TT, Evangelista A, Nienaber CA, et al. Partial thrombosis of the false lumen in patients with acute type B aortic dissection. $N$ Engl J Med. 2007;357:349-59.

41. Nienaber CA, Zannetti S, Barbieri B, et al. INvestigation of STEnt grafts in patients with type B Aortic Dissection: design of the INSTEAD trial - a prospective, multicenter, European randomized trial. $\mathrm{Am}$ Heart J. 2005;149:592-9.

42. Bozinovski J, Coselli JS. Outcomes and survival in surgical treatment of descending thoracic aorta with acute dissection. Ann Thorac Surg. 2008;85:965-70.

43. Eggebrecht H, Lonn L, Herold U, et al. Endovascular stent-graft placement for complications of acute type B aortic dissection. Curr Opin Cardiol. 2005;20:477-83. 
